\title{
Design an Identification Function to Reduce the Computational Resources on the Testing Process of an Analog Electronic Circuit
}

\author{
Sebastian Temich, Tomasz Golonek, Damian Grzechca \\ Faculty of Automatic Control, Electronics and Computer Science, Institute of Electronics, Silesian \\ University of Technology, \\ Akademicka 16, 44-100, Gliwice, Poland \\ damian.grzechca@pols.pl
}

\begin{abstract}
In modern electronic circuits, imperfectness in the technological process can cause errors in reaching the correct values of the functional parameters. In order to solve this problem, a novel approach of analog and mixed-signal circuit testing methodology is used. The presented approach allows the testing complexity to be reduced and the testing time to be decreased. For this paper, selected signal features were designated from the transient output signal response. Using regression models with the extracted signal features, the functional parameters of a circuit were determined. An evolutionary determination of the regression models enabled the efficiency of the identification process to be maximized. The proposed methodology is presented for an exemplary CMOS Dickson charge pump circuit.
\end{abstract}

Index Terms-Dickson charge-pump; Functional test; Incipient faults; Evolutionary regression; CMOS circuit identification.

\section{INTRODUCTION}

The rapid developments in electronic circuit design have not been accompanied by the same degree of progress in analog and mixed-signal electronic maintenance [1]. The standard production test approach for analog and mixedsignal circuits is based on measuring the functional parameters that are given on the data sheet and comparing them to the designed specifications in order to make the correct (go/no-go) decision [2]-[6]. Some specification parameters are measured directly, while others require a dedicated infrastructure in order to be measured [2], [7], [8].

The fault diagnosis methods for analog electronic circuits (AEC) are usually classified depending on whether a simulation is carried out before or after the testing [1]. The parameter identification techniques provide the values of all of the circuit parameters within a faulty circuit.

The internal circuit variables as well as the inputs and outputs are generally continuous and are difficult to quantize. The testability of analog circuits also depends on the component parametric deviations and the influence of environmental factors (temperature, electromagnetic

Manuscript received 23 September, 2018; accepted 4 January, 2019.

This work was supported by the Ministry of Science and Higher Education for statutory activities. interference, etc.) whose impact is very important [9].

A wide range of methods for testing the functional parameters of analog circuits have been proposed in the literature. An optimization formulation approach is a commonly used tool that is based directly on the circuit structure [9]. It is intuitive and proves to be effective on many occasions. Other approaches use neural networks [4], [10]-[12], classification methods that are based on a support vector machine, etc. [13]. In addition to neural networks, other artificial intelligence algorithms are also commonly used to test the specification parameters: genetic algorithms [13]-[15], fuzzy logic systems [16], dependency models [14], etc.

In the classical approach for testing analog or mixedsignal systems to determine the selected parameters of the design specification in order to check them in the go/no-go, several dedicated devices, several different stimulus signals and several modifications of the tested system (load, no load, etc.) can be used (Fig. 1). Such an approach, even if it is fully automated, requires a great deal of time to decide about go/no-go on the circuit. In the testing methodology proposed in this paper, the classical approach is replaced by using the regression of selected characteristics of the output signal, which allows selected functional parameters to be identified. The proposed method enables only one test signal and testing circuit to be used in only one configuration (no load) (Fig. 1). In the presented approach, the evolutionary determination of the terms of the approximate polynomial enables the estimation accuracy of the functional parameters of the system to be increased.

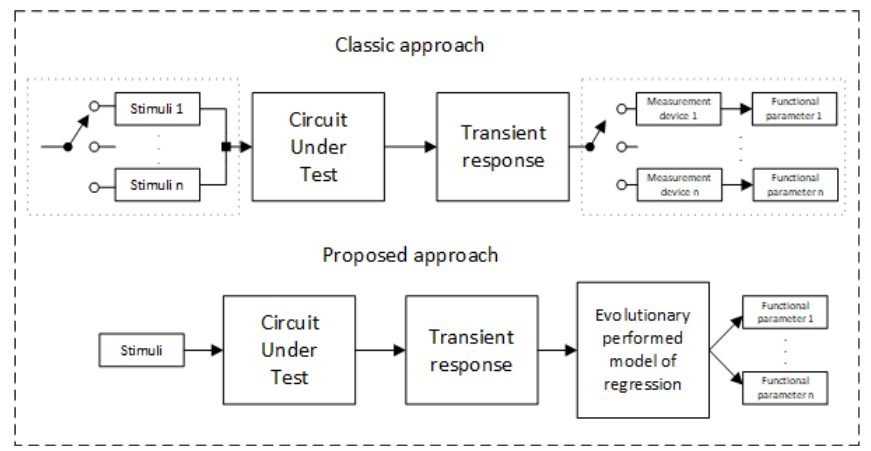
Fig. 1. The concept of the proposed test compared to the classical approach. 
Reducing the number of test signals enables the testing time of the system to be shortened, which is very important on a production line.

The presented method was implemented for a Dickson charge pump circuit constructed with CMOS technology for which production process errors were considered (incipient faults).

\section{A. Circuit under Test}

Charge pumps are power converters that adjust the power supply voltage to higher or lower constant voltages. Charge pumps transfer charge packets from the power supply to the output terminal using switches to generate the required voltage level. In micro-systems, charge pumps are usually fully built on-chip rather than off-chip. The Dickson charge pump circuit is presented in Fig. 2.

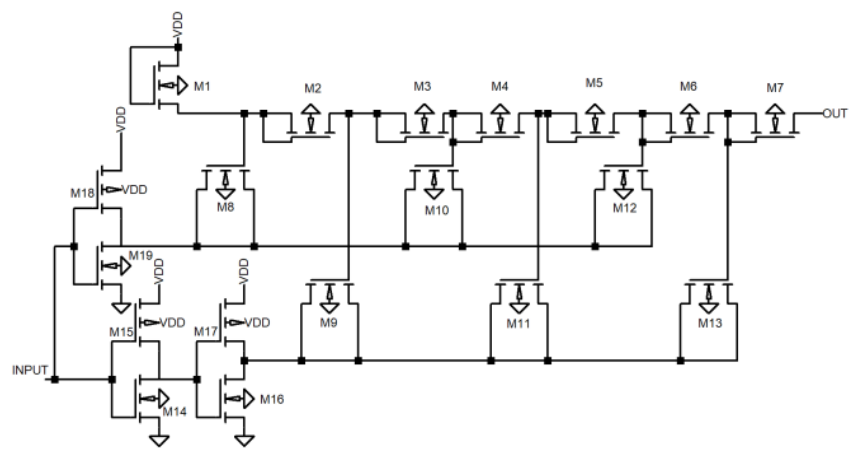

Fig. 2. Dickson charge pump circuit [18].

The functional identification of a Dickson charge pump should be focused on measuring the different parameters of the output signal for the selected stimuli [18]-[20]. In this research, the following functional parameters of the circuit being tested were analysed [18], [19], [21]:

$p_{1}$ - gain of the circuit;

$p_{2}$ - load-dependent losses (PLD) - measured with a $100 \mathrm{k} \Omega$ load;

$p_{3}$ - power consumption.

Table I presents the nominal values of the Dickson charge pump functional parameters.

TABLE I. NOMINAL VALUES OF THE DICKSON CHARGE PUMP SPECIFICATION PARAMETERS.

\begin{tabular}{|c|c|c|}
\hline $\boldsymbol{p}_{\mathbf{1}}[\mathbf{V} / \mathbf{V}]$ & $\boldsymbol{p}_{\mathbf{2}}[\mathbf{m W}]$ & $\boldsymbol{p}_{\mathbf{3}}[\mathbf{m W}]$ \\
\hline 1.08 & 1.28 & 4.57 \\
\hline
\end{tabular}

\section{B. Technological Process Imperfectness}

The circuit that was tested that is presented in this paper is based on MOS transistors (Fig. 2) for which incipient faults can occur at the production stage because of the imperfectness of the technological processes. The authors focused on the exemplary faults that are caused by the movement of the photolithographic mask, which changes the parametric values of the transistor structure - the length $(L)$ and width $(W)$ of a channel [13]. A change in the $W$ and $L$ of MOS transistors has a significant impact on the transistor specification parameters (i.e. the threshold voltage or channel length modulation parameter) [22], [23], which has affects the functional parameters of a Dickson charge pump.

In this research, the nominal values of length $L$ and width $W$ in MOS transistors are defined with $L_{n o m}, W_{n o m}$. The maximum range for the $L$ and $W$ parameters were set to:

$$
\left\{\begin{array}{l}
W \in<W_{\min } ; W_{\max }> \\
L \in<L_{\min } ; L_{\max }>
\end{array}\right.
$$

where $W_{\min } / W_{\max }$ and $L_{\min } / L_{\max }$ are calculated as:

$$
\left\{\begin{array}{l}
W_{\min } / L_{\min }=W_{\text {nom }} / L_{\text {nom }} \times 0.95 \\
W_{\max } / L_{\max }=W_{\text {nom }} / L_{\text {nom }} \times 1.05
\end{array}\right.
$$

In the entire range from $W_{\min } / L_{\min }$ to $W_{\max } / L_{\max }$, the parameters of the length and width of the transistor channel are calculated as

$$
W_{i} / L_{i}=W_{\text {nom }} / L_{n o m} \times \delta_{i},
$$

where $\delta_{\mathrm{i}} \epsilon\{0.95,0.96,0.97, \ldots, 1.05\}$.

The above change in the technological parameters of the transistors permits a precise determination of any changes in the selected functional parameters (performance) of a CUT as a function of incipient faults.

\section{Simulation of a CUT}

The Dickson charge pump was modelled using Spice software and the transistor models are represented by a BSIM numerical description [24].

The transistors from Fig. 2 are represented by the BSIM models of $50 \mathrm{~nm}$ technology; the $L$ and $W$ values are listed in Table II.

\begin{tabular}{|c|c|c|c|c|c|}
\hline & $\begin{array}{l}L \text { of the } \\
\text { channel }\end{array}$ & $\begin{array}{l}\text { W of the } \\
\text { channel }\end{array}$ & & $\begin{array}{l}L \text { of the } \\
\text { channel }\end{array}$ & $\begin{array}{l}\text { W of the } \\
\text { channel }\end{array}$ \\
\hline M1 & $50 n$ & $500 \mathrm{n}$ & M11 & $200 n$ & $200 \mathrm{n}$ \\
\hline M2 & $50 n$ & $100 \mathrm{n}$ & M12 & $200 n$ & $200 n$ \\
\hline M3 & $50 n$ & $100 \mathrm{n}$ & M13 & $200 n$ & $200 n$ \\
\hline M4 & $50 n$ & $100 \mathrm{n}$ & M14 & $50 n$ & $250 \mathrm{n}$ \\
\hline M5 & $50 n$ & $100 \mathrm{n}$ & M15 & $50 n$ & $500 n$ \\
\hline M6 & $50 n$ & $100 \mathrm{n}$ & M16 & $50 n$ & $250 \mathrm{n}$ \\
\hline M7 & $50 n$ & $100 \mathrm{n}$ & M17 & $50 n$ & $500 \mathrm{n}$ \\
\hline M8 & $200 \mathrm{n}$ & $200 \mathrm{n}$ & M18 & $50 n$ & $500 \mathrm{n}$ \\
\hline M9 & $200 n$ & $200 \mathrm{n}$ & M19 & $50 n$ & $500 \mathrm{n}$ \\
\hline M10 & $200 \mathrm{n}$ & $200 \mathrm{n}$ & & & \\
\hline
\end{tabular}

TABLE II. LENGTH AND WIDTH OF THE TRANSISTOR CHANNELS.

At the simulation stage, the CUT was stimulated using a pulse waveform that ranged from $0 \mathrm{~V}$ to $3.3 \mathrm{~V}$ with a rising time $t_{r}=10 \mathrm{ps}$ a falling time $t_{f}=t_{r}$ and a period time $T_{\text {period }}=$ $20 \mathrm{~ns}$, which were delivered to the 'INPUT'. In addition, the circuit was sourced by a 3.3 VDC that was delivered to the 'VDD' as is illustrated in Fig. 3.

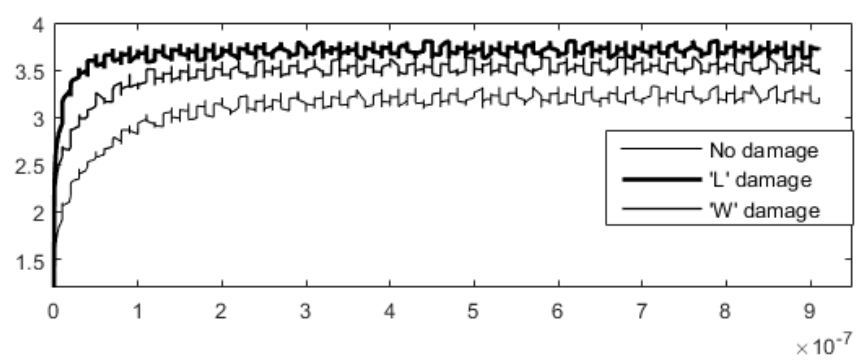

Fig. 3. Exemplary responses of the Dickson charge pump circuit.

In accordance with the assumptions of the SBT method [14], the presented circuit, along with any subsequent 
failures was simulated once, and all of the data that was collected during the simulation process formed the basis for further analysis using the presented method.

\section{Output Signal Feature Selection}

The main goal of the approach that is presented in this paper is to shorten the test time while simultaneously maximizing the identification accuracy using the evolutionary determined regression model. For this purpose, the $N=7$ signal features $\left(x_{1}, \ldots, x_{7}\right)$ from the $u_{\text {out }}(t)$ for the selected stimuli $u_{i n}(t)$ were designated. The designated features were [25], [26]:

1. Rising time of the $u_{\text {out }}(t)\left(T_{R}\right)-$ Time that the $u_{\text {out }}(t)$ required to rise from $10 \%$ to $90 \%$ of the steady-state response;

2. The peak value of the $u_{\text {out }}(t)\left(P e a k_{\text {out }}\right)$;

3. Settling time of the $u_{\text {out }}(t)\left(S t_{\text {out }}\right)$ - The time after which the ripples of the $u_{\text {out }}(t)$ are less than $2 \%$;

4. A variance of the $u_{\text {out }}(t)$ (Var out $)$ from 0 to the settling time of the $u_{\text {out }}(t)$;

5. Settling minimum $\left(S_{\text {min }}\right)$ value of the $u_{\text {out }}(t)$;

6. Settling maximum value $\left(S_{\max }\right)$ of the $u_{\text {out }}(t)$;

7. Mean value of the span of the $u_{\text {out }}(t)\left(M_{\text {out }}\right)$, calculated as

$$
M_{\text {out }}=\frac{S_{\max }+S_{\min }}{2} .
$$

The selected features of the $u_{\text {out }}(t)$ created a feature vector $\boldsymbol{X}$ for each of the simulated cases

$$
\mathbf{X}=\left[T_{R}, \text { Peak }_{\text {out }}, S t_{\text {out }}, \text { Var }_{\text {out }}, S_{\min }, S_{\max }, M_{\text {out }}\right],
$$

on the basis of which a regression model to identify the selected functional parameters of the Dickson charge pump was created.

\section{E. Time Analysis}

In addition to determine the most accurate mapping of the functional parameters of an AEC, the time of the test should also be as short as possible.

The overall time of a test is equal to the sum of the times of the individual stages. In the classical approach, this is the time required to analyse the selected parameters of the $u_{\text {out }}(t)$ using external measuring devices, to modify the circuit to enable the measurement of the duty parameter that is characteristic for a given work point, etc. In the presented approach, the test time ( $\left.T_{\text {overall }}\right)$ was equal to the sum of the times of all of the subsequent steps: the time required to determine all the features of the $u_{\text {out }}(t)-T_{f}$ and the time required to identify selected functional parameters $T_{p 1}, T_{p 2}$, $T_{p 3}$

$$
T_{\text {overall }}=T_{f}+T_{p 1}+T_{p 2}+T_{p 3} .
$$

The AEC that was used to verify the correctness of the test specification methodology is presented in Fig. 4. The analog circuit response signature $u_{\text {out }}(t)$ was acquired directly after its stimulation during the test stage using a dedicated signal $u_{i n}(t)$. Typically, a step or pulse stimulus as well as square waveform can be applied to the AEC to test the excitation. These kinds of signals can be generated precisely in a simple way and they ensure rich spectra of the frequency components that will explore the signal path of the tested circuit well.

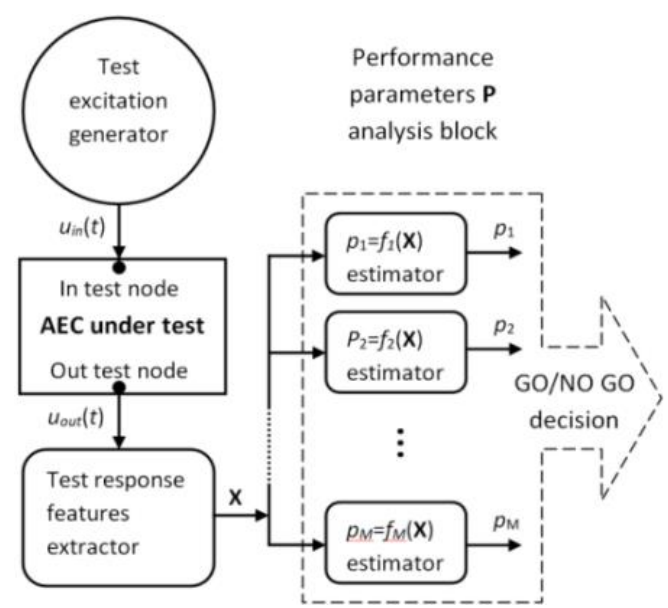

Fig. 4. The analog electronic circuit specification met the fulfillment test.

The set of assumed features $\mathbf{X}$ of the test signature $u_{\text {out }}(t)$ was used to explain the actual levels of the tested performance parameters $p_{1}, p_{2}, \ldots, p_{M}$ using the respective mathematical formulas (models).

\section{ANALOG ELECTRONIC CIRCUIT SPECIFICATION TESTING}

A crucial point of the proposed testing methodology is determining the most appropriate $M$ mathematical models, i.e. the functions $f_{1}(\mathbf{X}), f_{2}(\mathbf{X}), \ldots, f_{M}(\mathbf{X})$ that will ensure precise and quick estimations of the tested performance parameters of an AEC, i.e. at a low cost and less timeconsuming laboratory measurements. In the proposed solution, these models are non-linear ones and they have the structure of the sum of $K$-th order regression polynomials, which are explained in detail in Section II. The coefficients (terms) of these polynomials were searched before test stage in the dedicated evolutionary system that is described in Section III. For the proposed approach, the main computational effort is only required before the exact testing (i.e. only at the before test stage) during the evolutionary search for the mathematical models. However, at the specification test stage, they are known and the mathematical estimators that are found ensure a highquality, low-cost and very quick procedure. Finally, when all of the parameters that have been estimated in this way fulfill the assumed design restrictions (specification), then a verified AEC successfully passes the test or it is determined to be faulty (go/no go decision). Another advantage of this technique is the full identification of the complete set of the tested parameters. This property permits the automatic classification of the AECs that are produced to the quality classes according to the performances are actually achieved.

\section{STRUCTURE OF THE REGRESSION MODELS}

The proposed polynomial regression models are mathematical relationships between the assumed features (collected in vector $\mathbf{X}$ ) of the registered test signature $u_{\text {out }}(t)$ and the set $\mathbf{P}$ of the $M$ performance parameters of the AEC that is to be verified

$$
\mathbf{P}=\left[p_{1}, p_{2}, \ldots, p_{M}\right]
$$


This kind of relationship, which is designated during the before test computations, permits an AEC tester that is oriented to the verification of the specification using the precisely estimated actual values of the performance parameters that are observed at the testing stage to be created. The main advantage of this concept is the possibility of an easy and quick specification testing of the realization of an AEC using a low-cost tester that can be implemented in a mixed-signal system in a simple way. This capability also makes it possible to reach the build in selftesting (BIST) procedure of the controlled electronic system.

The general mathematical formula of the proposed regression model that was created to estimate the $m$-th ( $m=$ $1, \ldots, M)$ AEC performance parameter is

$$
p_{m}(\mathbf{X})=\sum_{n=1}^{N} \sum_{k=1}^{K} a_{m n k} \times x_{n}^{k}+b_{m}
$$

where $\mathbf{X}$ is the vector that consists of the $N$ features that were extracted from the time test signature (defined initially to the investigated parameter $p_{m}$ explanation)and $a_{m n k}$ and $b$ are the variable elements and constant terms of the $K$-th order approximation polynomial, respectively. During the AEC testing stage, the time response to the assumed testing excitation is acquired only once and its selected $N$ features (such as the rise time, overshoot, steady-state level) create the explanatory variable vector $\mathbf{X}$

$$
\mathbf{X}=\left[x_{1}, x_{2}, \ldots, x_{N}\right]
$$

The elements of (9) can be defined based on an engineer's experience and knowledge about the classes of the explained performance parameters (e.g. step response rise time is correlated to the bandwidth well and the steady-state DC voltage level describes the DC gain of the tested AEC well); they can be also selected experimentally. As can easily be noticed from the expanded form of the model (10)

$$
\begin{gathered}
p_{m}(\mathbf{X})=a_{m 1 K} \times x_{1}^{K}+\ldots+a_{m 12} \times x_{1}^{2}+a_{m 11} \times x_{1}^{1}+ \\
+a_{m 2 K} \times x_{2}^{K}+\ldots+a_{m 22} \times x_{2}^{2}+a_{m 21} \times x_{2}^{1}+ \\
\vdots \\
+a_{m N K} \times x_{N}^{K}+\ldots+a_{m N 2} \times x_{N}^{2}+a_{m N 1} \times x_{N}^{1}+b_{m}
\end{gathered}
$$

Each element of the explanatory variables that are collected in (9) is an argument of the dedicated individual polynomial that is a part of the complex formula that is able to model strongly non-linear relationships. This property makes it possible to create mathematical equations respective to describing tested parameter $p_{m}$ in a wide range of variations. The above model (10) must be defined independently for all of the diagnosed $M$ performance parameters, so that finally, the tester is completely oriented to the requirements of the specification validation

$$
L=M \times(N \times K+1),
$$

coefficients where $N$ and $K$ are the power of set (9) and the assumed order of the polynomials, respectively.

\section{EVOlutionary SEARCHING FOR OPTIMAL MODELS}

The evolutionary computations have been applied to many difficult tasks successfully [5], [15], [27]. Although these kinds of calculations are heuristic, they are a powerful tool for probing and searching a huge data space for the most appropriate solution of problems that are tedious for performing deterministic analysis in a reasonable time. The evolutionary system that is described in this paper applies differential evolution (DE) to the multidimensional equations (9) determination [28]. The genotype structure and evolutionary computation scheme are illustrated in Fig. 5. The optimization of the regression polynomial coefficients proceeds in a population of $I$ individuals, which keep $(N \cdot K+1)$ real numbered values. During the start (primary) generation creation, the linear section terms of (10) (i.e. $a_{m 11}, \ldots, a_{m N 1}$ and $b_{m}$ ) of the first genotype are initialized to the values that were obtained from the standard algorithm of linear multiple regression, respectively [28]. However, the remaining terms (i.e. for the squared and higher order variables) are initialized with respective values that are dispersed randomly from those that were calculated as linear ones. This solution enables a good start point for the exploring the search space to be defined and, as a result, it improves the convergence to an optimal solution speed. After completing the primary population genes, the fitness value $Q_{m}$ is calculated for each one. The coefficient of determination (squared R) is applied in the designed evolutionary system as a measure of the quality of the searched relationships (phenotype) that were determined

$$
Q_{m}=R_{m}^{2}=1-\frac{\sum_{j=0}^{J-1}\left(p_{m j}-\hat{p}_{m j}\right)^{2}}{\sum_{j=0}^{J-1}\left(p_{m j}-\bar{p}_{m}\right)^{2}},
$$

where $m$ is the index of the tested AEC performance parameter, $p_{m j}, \hat{p}_{m j}$ are its accurate, estimated (obtained from the evaluated formula) values and $\bar{p}_{m}$ is the average value of the parameter in the set of $J$ training patterns.

The nominator and denominator in function (12) are the sum of squared errors and the total sum of squares that were calculated in the training set. This function is a standard measure of fitness between the analysed function and the statistical data, which reaches a unit value for the ideally defined relationship using an analytical form of the mathematical model. Next, when the primary population is ready, the DE algorithm is run for the $T$ generations for which each offspring genotype is created in the way that is illustrated in Fig. 2. In order to obtain a new individual, the processed $i$-th parental genotype $\mathbf{G}_{i}$ is crossovered with $\tilde{\mathbf{G}}_{i}$ one that was obtained after the recombination of the differential material

$$
\tilde{\mathbf{G}}_{i}=\mathbf{G}_{i}+w \times\left(\mathbf{G}_{b t}-\mathbf{G}_{i}\right)+(1-w) \times\left(\mathbf{G}_{r 1}-\mathbf{G}_{r 2}\right),
$$

where $\mathbf{G}_{b t}, \mathbf{G}_{r 1}, \mathbf{G}_{r 2}$ are the best ones that were found during all of the previous iterations and two randomly selected genotypes; however, $w$ is the weight that balances the 
recombination levels from the specific parts. During the crossover operation, the original genes can be replaced by the new ones (i.e. with a 0.5 probability), which is calculated as the arithmetic average values between the genes of $\boldsymbol{G}_{\boldsymbol{i}}$ and $\tilde{\mathbf{G}}_{i}$, respectively. This procedure allows some of capabilities of the best currently known solution as well as some of the new characteristics from the random ones to be inherited (a kind of mutation process). The last step is the selection of better evaluated genotype code for the child, i.e. preserved, the original one (without any modifications) or the one that was obtained after the crossover, which can be added to the offspring population, respectively. When the whole newly gathered generation is ready, it replaces the old one during succession. Finally, after $T$ cycles of the evolutionary system, the best found genotype code vectors of the coefficients are ready to estimate the tested parameter with the highest score level (12).

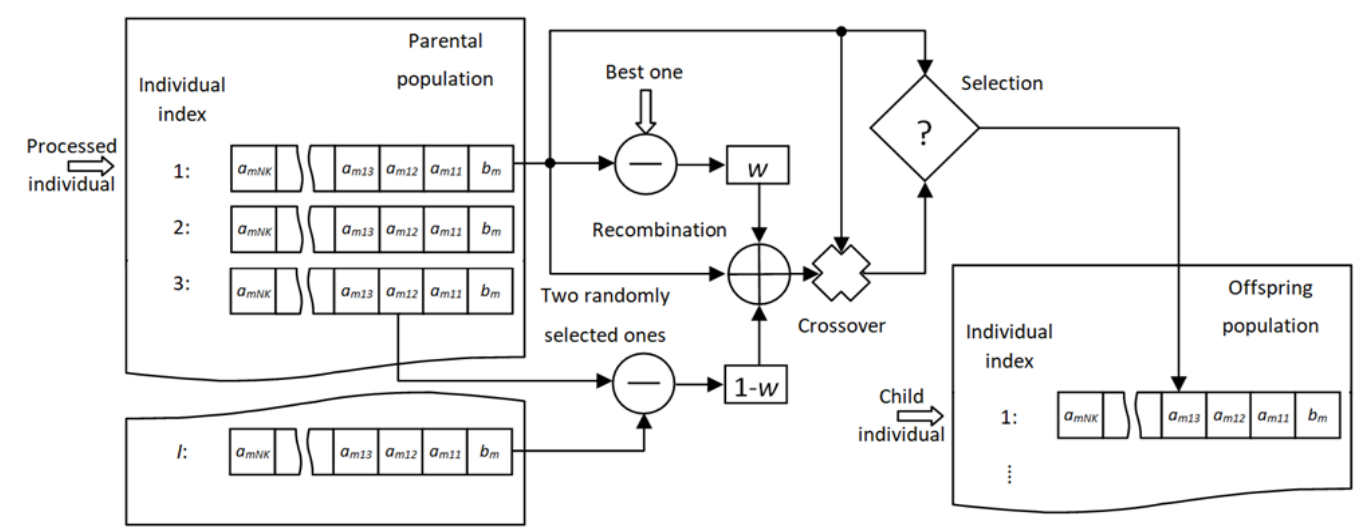

Fig. 5. Diagram of the evolutionary system cycle.

The training patterns that are used to determine the fitness function are the simulation results of the tested AEC, which are obtained after Monte Carlo analysis of the circuits with the assumed perturbations (e.g. with the dimensions of the structures of integrated on a silicon die elements dispersed randomly), which imitate the production or post-production defects of the circuit. This set of $J$ before test analysis results defines the signatures of the AEC with various levels of the observed performance parameters $p_{m}(m=1, \ldots, M)$, which makes it possible to investigate the statistical relationships between them and the delegated easily measurable explanatory features of the time response signature. Besides, it should be emphasized that the all of the simulation calculations are executed during the testing formula (8) designing stage only; however, the resulting specification test requires only $L-1$ multiplications and $L$ summations, respectively, i.e. the test computational cost is low and is related to (11).

\section{V.RESEARCH RESULTS}

The following section presents the results of the proposed method for identifying parameters. All of the simulations were performed on a computer with the following specifications: Intel Core i5 $3.2 \mathrm{GHz}$ with $8 \mathrm{~GB}$ RAM. For identifying all of the parameters, the $3^{\text {rd }}$ order polynomial was used. According to (11): $K=3, N=7$ and $M=3$, the evolutionary calculations were started for $T=2000$ iterations and $I=200$ individuals in the population. Searching for each mathematical formula (10) using the specialized terms to identify a specific tested parameter took about 20 minutes.

After identifying the selected functional parameters, a $\delta_{\mathrm{i}}$ range of the correct parameter tolerance was imposed on each of the $\mathrm{p}_{\mathrm{i}}$. This process allowed the accuracy of classification for each of the functional parameters of the presented method to be determined. The described method was also compared to the support vector regression model (SVR) with a Gaussian kernel function [28].

\section{A. Gain Parameter}

The first functional parameter for the proposed algorithm to identify was the gain parameter. By analysing the changes in parameters $L$ and $W$ of the transistor, the linear dependence of the effect of any damage to the photolithographic mask in a tolerance range equal to $5 \%$ of the value of the parameter $p_{1}$ should be observed. In connection with the above, for a value of parameter $p_{1}$ inside the tolerance range, a correlation coefficient was determined and it was equal to $98.72 \%$.

In the next stage, a $3^{\text {rd }}$ order polynomial was created to increase the identification efficiency. For the gain parameter, the determination of (10) error was equal to $\mathrm{Err}$ $=0.08 \%$.

The time required to obtain the identify parameter was equal to $T_{p l}=1.87 \mu \mathrm{s}$.

In accordance with (10), 22 coefficients were determined for $3^{\text {rd }}$ order polynomial. Each of their values is presented in (14):

$$
\left\{\begin{array}{l}
b_{0}=410, \\
a_{z i}=\{0.02,-7394,0.31 ;-0.0033,0,-2221 \\
0,-2605,-3016 ;-0.40,7 e^{-3}, 0 ; 1424,0,2380 \\
\left.4546,0.47,3 e^{-4} ; 0,-702.4,0\right\} .
\end{array}\right.
$$

Table III presents the exemplary values of $\mathrm{p}_{1}$ that were calculated by the proposed algorithm compared to the expected values.

Figure 6 presents the differences between the calculated and expected value of the gain functional parameter for the length deviation. As can be seen, the differences between the expected and calculated values are almost unnoticeable. 
TABLE III. EXEMPLARY VALUES OF THE EXPECTED AND CALCULATED GAIN SPECIFICATION PARAMETER.

\begin{tabular}{|c|c|}
\hline \multicolumn{2}{|c|}{$\begin{array}{c}\text { Exemplary Difference between the expected and calculated gain } \\
\text { value }\end{array}$} \\
\hline Expected & Calculated \\
\hline 1.1700 & 1.1647 \\
\hline
\end{tabular}

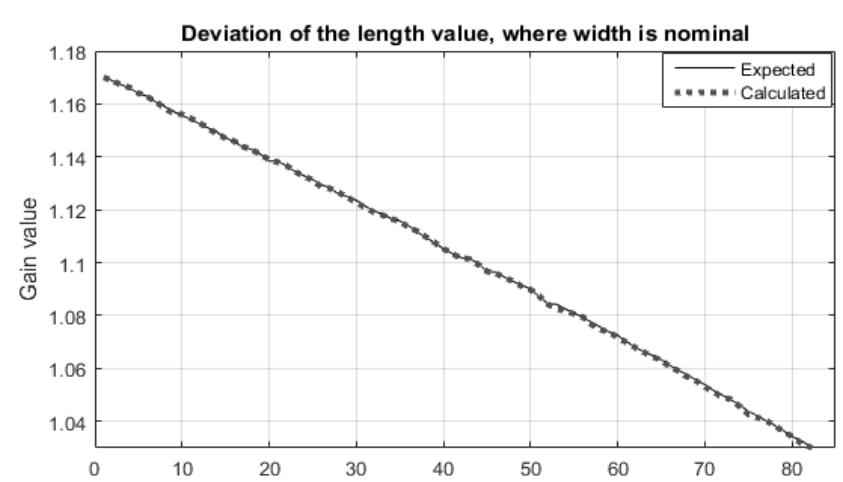

Fig. 6. Difference between the calculated and the expected gain parameter for the length deviation of the photolithographic mask.

The correlation between the obtained identification and correct identification is presented in Fig. 7. The differences between the respective values were unnoticeable.

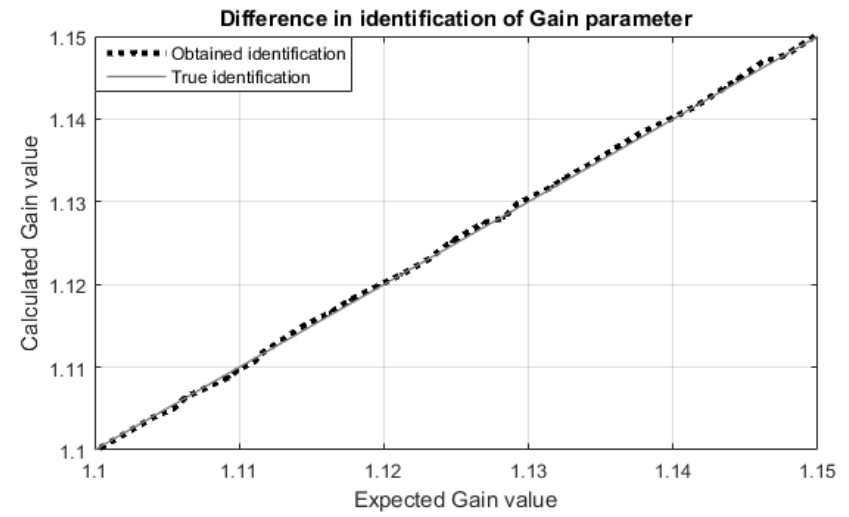

Fig. 7. Correlation between the correct and obtained identification of the gain parameter.

Because of the classification process, the nominal value of the $\mathrm{p}_{1}$ parameter for an undamaged circuit is equal to Gain $_{\text {Nom }}=1.08$ V/V. By imposing a $\delta_{\mathrm{i}}=2 \% \mathrm{p}_{1}$ tolerance range, the error matrix and sensitivity values for the proposed method were determined. Proposed method was compared to the SVR method. The results are presented in Table IV. The maximum error (for $3^{\text {rd }}$ order polynomial) of identification gain parameter inside tolerance range was equal to $0.16 \%$.

TABLE IV. CONTINGENCY TABLE WITH SENSITIVITY VALUE FOR P1.

\begin{tabular}{|c|c|c|c|}
\hline \multicolumn{4}{|c|}{ Error matrix } \\
\hline \multicolumn{2}{|c|}{ Proposed method } & \multicolumn{2}{c|}{ SVR } \\
\hline \multicolumn{2}{|c|}{ True positive } & \multicolumn{2}{c|}{ False negative } \\
\hline 2449 & 2394 & \multicolumn{2}{c|}{73} \\
\hline \multicolumn{2}{|c|}{ Sensitivity [\%] } \\
\hline \multicolumn{2}{|c|}{98.73} & \multicolumn{2}{|c|}{97} \\
\hline
\end{tabular}

\section{B. Load-dependent Losses}

The second functional parameter to identify was the loaddependent losses. Similar to the $p_{1}$ functional parameter, for the $\mathrm{p}_{2}$, changes in parameters $L$ and $W$ of the transistor have a linear dependence on the effect of damage to the photolithographic mask in a tolerance range equal to $5 \%$.
The correlation coefficient that was determined for $p_{2}$ was equal to $97.78 \%$. Next, a $3^{\text {rd }}$ order polynomial was created to increase the identification efficiency. For this parameter, the determination error of relationship (10) was equal to Err $=0.09 \%$.

The time required to obtain the $p_{2}$ was equal to $T_{p 2}=$ $1.5 \mu \mathrm{s}$. In order to determine parameter $\mathrm{p}_{2}$ in the classical approach, it is necessary to modify the AEC by adding an appropriate load to the circuit output. This treatment extends the time of testing the $p_{2}$ by the time that is required for an additional stimulation $u_{i n}(t)$, and an additional extraction of the features of $u_{\text {out }}(t)$. This time may increase up to two-fold compared to the described method.

In accordance with (9), (15) presents the polynomial coefficients:

$$
\left\{\begin{array}{l}
b_{0}=-0.725 \\
a_{z i}=\left\{-1 e^{-4},-26.95,6 e^{-5} ; 1.7 e^{-5}, 0,4.44\right. \\
0,-0.58,32.82 ;-5 e^{-4},-5 e^{-7}, 0 ;-2.16,0,-0.15 \\
\left.-40.28,2.5 e^{-5}, 2.3 e^{-5} ; 0,4.73,0\right\} .
\end{array}\right.
$$

Figure 8 presents the differences between the calculated and expected values of the load-dependent functional parameter for the length deviation of an incipient fault. The difference between these two values was as small as the gain parameter and the deviations were also minor.

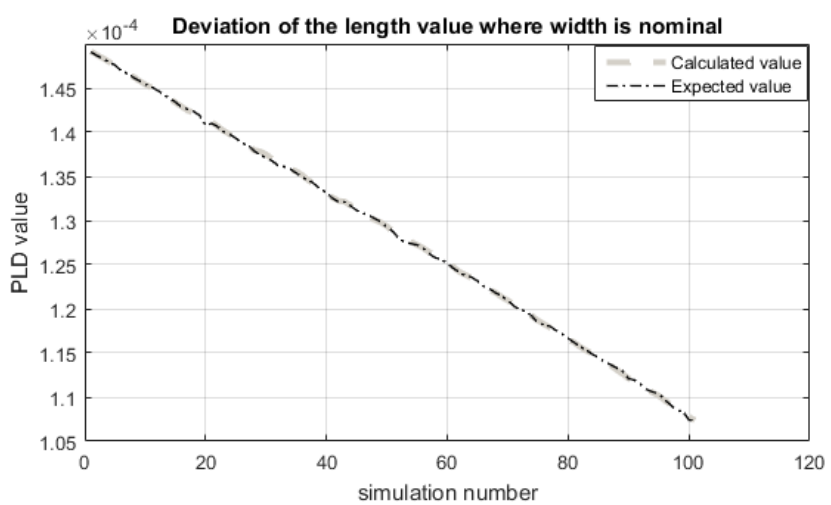

Fig. 8. Differences in the calculated and expected values of the loaddependent loss parameter for the length deviation of the photolithographic mask.

The correlation between the obtained identification and correct identification is presented in Fig. 9. As the figure shows, the differences between the respective values were minimal.

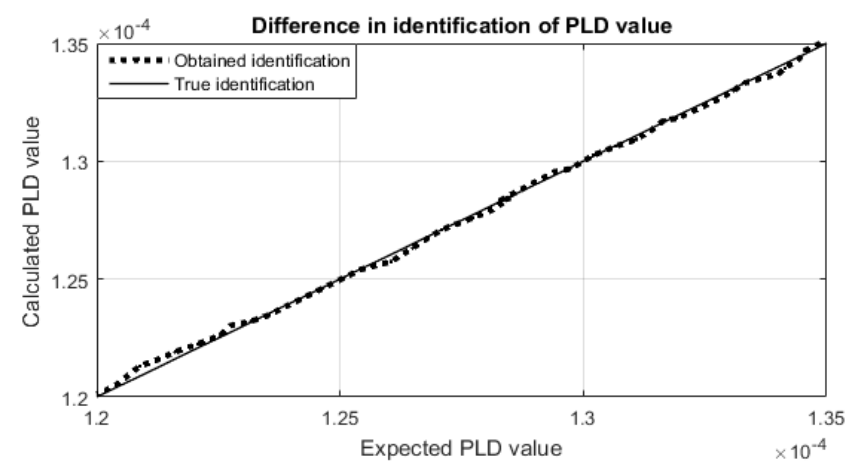

Fig. 9. Correlation between the correct and obtained identification of the $\mathrm{p}_{2}$ parameter. 
Because of the classification process, the nominal value of the PLD parameter for an undamaged circuit was equal: $P L D_{\text {Nom }}=4.57 \mathrm{~mW}$. By imposing a $\delta_{\mathrm{i}}=2 \% \mathrm{p}_{2}$ tolerance interval, a contingency table and sensitivity value for the proposed method were determined. The results with comparison to the SVR method are presented in Table V. The maximum error of $\mathrm{p}_{2}$ identification inside the tolerance range was equal to $16 \%$.

TABLE V. CONTINGENCY TABLE WITH THE SENSITIVITY VALUE FOR $P_{2}$.

\begin{tabular}{|c|c|c|c|}
\hline \multicolumn{3}{|c|}{ Error matrix } \\
\hline \multicolumn{2}{|c|}{ Proposed method } & \multicolumn{2}{c|}{ SVR } \\
\hline \multicolumn{2}{|c|}{ True positive } & \multicolumn{2}{c|}{ False negative } \\
\hline 1258 & 1254 & 11 & 15 \\
\hline \multicolumn{2}{|c|}{ Sensitivity [\%] } \\
\hline 99.13 & \multicolumn{2}{|c|}{98.82} \\
\hline
\end{tabular}

\section{Power Consumption}

The third functional parameter to identify was power consumption. Similar to the previous functional parameters, the linear dependence of the effect of damage to the photolithographic mask in a tolerance range equal to $5 \%$ for $L$ and $W$ were determined. The determined correlation coefficient of $\mathrm{p}_{3}$ was equal to $88.8 \%$. Next, a $3^{\text {rd }}$ order polynomial was created to increase the identification efficiency. For this parameter, the error in the determination was equal to $E r r=1.06 \%$.

The time required to obtain $\mathrm{p}_{3}$ was equal to $T_{p 3}=1.78 \mu \mathrm{s}$.

In accordance with (9), (16) presents polynomial coefficients:

$$
\left\{\begin{array}{l}
b_{0}=661.4 \\
a_{z i}=\left\{4.195,-2642,0.1 ; 2.6 e^{-3}, 0,2328\right. \\
0,-4672,-7739 ; 1.7 e^{-3}, 0.6 e^{-3}, 0 ; 1127,0,-398.4 \\
\left.-2928,-0.013,6 e^{-3} ; 0,-223.1,0\right\}
\end{array}\right.
$$

Table VI presents the exemplary values of $\mathrm{p}_{3}$ that were calculated using the proposed algorithm compared to the expected values.

TABLE VI. EXEMPLARY VALUES OF THE EXPECTED AND CALCULATED.SPECIFICATION PARAMETER.

Exemplary difference between the expected and calculated power

\begin{tabular}{|c|c|}
\hline $\begin{array}{c}\text { Exemplary difference between the expected and calculated power } \\
\text { consumption value }\end{array}$ \\
\hline Expected & Calculated \\
\hline $4.61 \mathrm{~mW}$ & $4.60 \mathrm{~mW}$ \\
\hline
\end{tabular}

Figure 10 presents the difference between the calculated and expected value of the power consumption functional parameter for the width deviation of the photolithographic mask. The difference between these two values is not as small as for the gain parameter but the deviations were also minor.

The correlation between the obtained identification and correct identification is presented in Fig. 11. As the figure shows, the differences between the respective values were noticeable and an offset error was observed for the analysed cases.

Because of the classification process, the nominal value of the $p_{3}$ parameter for an undamaged circuit was equal: PDisp Nom $_{\text {Nom }}=1.28 \mathrm{~mW}$. By imposing a $\delta_{\mathrm{i}}=0.5 \% \mathrm{p}_{3}$ tolerance interval, a contingency table and sensitivity value for the proposed method were. The results with comparison to the SVR method are presented in Table VII. The maximum error of $\mathrm{p}_{3}$ identification inside the tolerance range was equal to $0.802 \%$. In accordance with (12), Table VIII presents $Q_{m}$ value of the identification process.

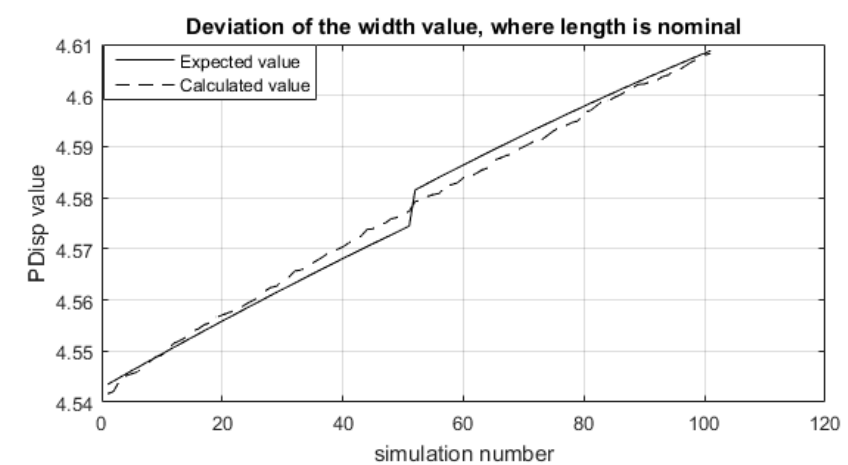

Fig. 10. Difference between the calculated and expected power consumption parameter for the width deviation of the photolithographic mask.

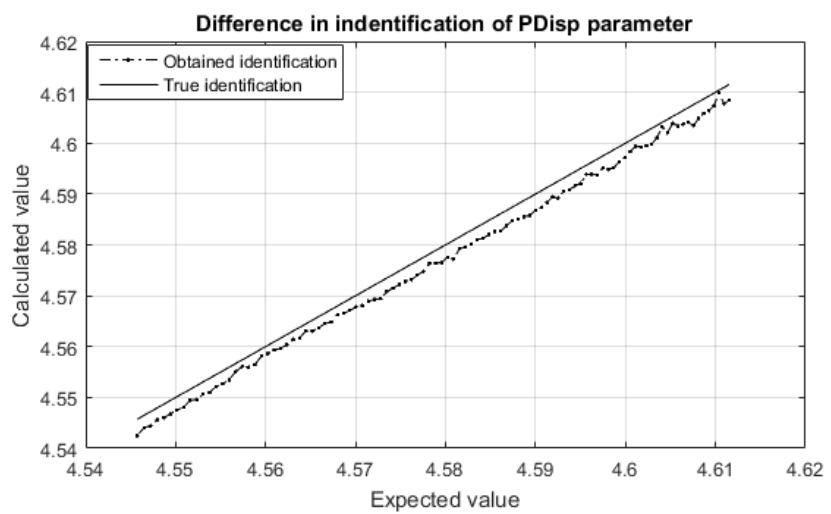

Fig. 11. Correlation between the correct and obtained identification of the $\mathrm{p}_{3}$ parameter.

TABLE VII. CONTINGENCY TABLE WITH THE SENSITIVITY VALUE FOR $P_{3}$

\begin{tabular}{|c|c|c|c|}
\hline \multicolumn{4}{|c|}{ Error matrix } \\
\hline \multicolumn{2}{|c|}{ Proposed method } & \multicolumn{2}{|c|}{ SVR } \\
\hline \multicolumn{2}{|c|}{ True positive } & \multicolumn{2}{|c|}{ False negative } \\
\hline 6834 & 6843 & 253 & 231 \\
\hline \multicolumn{4}{|c|}{ Sensitivity [\%] } \\
\hline \multicolumn{2}{|c|}{96.43} & \multicolumn{2}{|c|}{96.73} \\
\hline
\end{tabular}

TABLE VIII. EXEMPLARY VALUES OF THE EXPECTED AND CALCULATED SPECIFICATION PARAMETER.

\begin{tabular}{|c|c|}
\hline Specification parameter & $\begin{array}{c}\text { Coefficient of the determination } \\
\text { value [\%] }\end{array}$ \\
\hline Gain & 99.92 \\
\hline Load dependent losses & 99.91 \\
\hline Power consumption & 98.94 \\
\hline
\end{tabular}

Table IX presents the time required to complete the individual steps of the presented method.

TABLE IX. TIME VALUES OF THE COMPONENTS OF THE PRESENTED METHOD.

\begin{tabular}{|c|c|}
\hline & $\begin{array}{c}\text { Time to determine each of the } \\
\text { elements }\end{array}$ \\
\hline$T_{f}$ & $9 \mathrm{~ms}$ \\
\hline$T_{p 1}$ & $1.87 \mu \mathrm{s}$ \\
\hline$T_{p 2}$ & $1.5 \mu \mathrm{s}$ \\
\hline$T_{p 3}$ & $1.78 \mu \mathrm{s}$ \\
\hline$T_{\text {overall }}$ & $9.05 \mathrm{~ms}$ \\
\hline
\end{tabular}

In the classical approach, for determining the value of $p_{2}$, it is necessary to modify the CUT with an additional 
element and to stimulate it with $u_{\text {in }}(t)$ once again. The features of vector $\mathbf{X}$ also have to be appointed. In this case, the time for test using the classical approach is equal to $T_{\text {overall }}=T_{\text {overall }}+T_{f}+T_{p 2}$ and is twice as long as in the proposed method.

\section{D.Proposed Method Efficiency Testing}

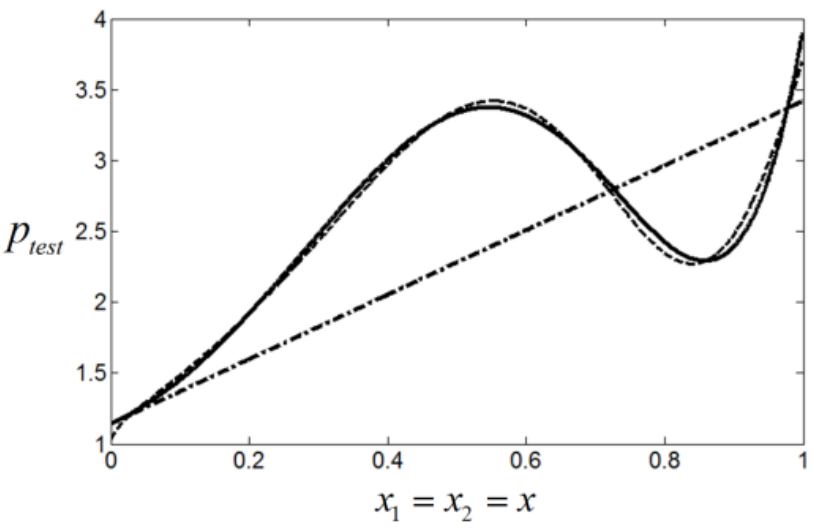

Fig. 7. Results of the exemplary, benchmark function optimization.

Optimization convergence of nonlinear mathematical model by means of the proposed evolutionary technique may be confirmed by a simple experiment. The exemplary test function characterized with strong nonlinear relation between explanatory and explained variables may be searched within wide range of their arguments variation. For this experiment purpose, the representative, exemplary function was delegated

$$
p_{\text {test }}\left(x_{1}, x_{2}\right)=e^{x_{1}}+\sqrt{x_{2}}+\sin \left(2 \times \pi \times x_{1} \times x_{2}\right) .
$$

Next, the algorithm described in this paper was started with $I=200$ individuals in population for $T=2000$ iterations. The 200 values of arguments $x_{1}=x_{2}=x$ dispersed randomly with uniform probability in assumed probing range $\langle 0,1\rangle$ were sampling tested function (17). The effects of approximation with $9^{\text {th }}$ ordered model (10) of the gathered points may be seen in Fig. 12, where dashed and solid lines curves are precise and estimated ones respectively. Concluding, the function determined by multiple linear regression (dash-dotted line) has quite low determination coefficient 0.543 of the tested reference one, however the found nonlinear model is very well fitted with 0.998 value of quality (12).

\section{CONCLUSIONS}

A specification-driven testing approach is proposed in this paper. Multiple regressions supported by evolutionary computations were used to identify the selected functional performance of a Dickson charge pump circuit.

The selection of the appropriate features of the signal circuit response $u_{\text {out }}(t)$ to the proposed stimuli $u_{\text {in }}(t)$ enabled the time required to test the signature analysis to be shortened: The time needed to obtain the last feature of the $\mathrm{u}_{\text {out }}(\mathrm{t})$ is equal about $0.7 \mu \mathrm{s}$ and the overall time needed to identify all selected functional parameters value is equal $9.05 \mathrm{~ms}$. Proposed methodology allow to identify 3 functional parameters of the CUT (gain, load-depend losses and power consumption), with $98 \%$ of the average sensitivity inside the tolerance range.

The proposed methodology is easy to implement on a production line. The polynomials, which were created using a regression algorithm, consist of only multiplications and additions, which enables the basic circuits of microcontrollers to be used.

\section{REFERENCES}

[1] S. D. Djordjevic, "Analog circuit diagnosis based on the nullor concept and multiport description of the circuit", Analog Integrated Circuits and Signal Processing, vol. 95, no. 1, pp. 141-149, 2018. DOI: $10.1007 / \mathrm{s} 10470-018-1123-7$

[2] S. D. Djordjevic, "Analog circuit diagnosis based on the nullor concept and multiport description of the circuit", Analog Integrated Circuits and Signal Processing, vol. 95, no. 1, pp. 141-149, 2018. DOI: 10.1109/TCAD.2017.2783302.

[3] M. J. Barragan, H.-G. Stratigopoulos, S. Mir, H. Le-Gall, N. Bhargava, A. Bal, "Practical simulation flow for evaluating analog/mixed-signal test techniques", IEEE Design \& Test, vol. 33, no. 6, pp. 46-54, 2016. DOI: 10.1109/MDAT.2016.2590985.

[4] S. Temich, D. Grzechca, "Application of neural network for testing selected specification parameters of voltage-controlled oscillator", Int J. Electron. Telecommun., vol. 64, no. 2, pp. 203-207, 2018. DOI: $10.24425 / 119371$

[5] T. Golonek, J. Machniewski, "Analog circuit specification testing by means of Walsh-Hadamard transform and multiple regression supported by evolutionary computations", Circuits Syst. Signal Process., vol. 37, no. 7, pp. 2736-2771, 2018. DOI: 10.1007/s00034017-0708-1.

[6] L. Chruszczyk, "Automatic test bench for selected transmission parameters of power line conductors", Int. J. Electron. Telecommun., vol. 61, no. 1, pp. 59-65, 2015. DOI: 10.1515/eletel-2015-0008.

[7] M. Tadeusiewicz, S. Halgas, "Diagnosis of soft spot short defects in analog circuits considering the thermal behaviour of the chip", Metrol. Meas. Syst., vol. 23, no. 2, 2016. DOI: 10.1515/mms-20160023.

[8] S. Halgas, M. Tadeusiewicz, "Improvement of the search method for parametric fault diagnosis of analog integrated circuits", in Proc. 23rd Int. Conf. Mix. Des. Integr. Circuits Syst. Mix., 2016, pp. 359-362. DOI: 10.1109/MIXDES.2016.7529765.

[9] L. Ji, X. Hu, "Analog circuit soft-fault diagnosis based on sensitivity analysis with minimum fault number rule", Analog Integr. Circuits Signal Process., vol. 95, no. 1, pp. 163-171, 2018. DOI: 10.1007/s10470-018-1111-y.

[10] Y. Deng, Y. Shi, W. Zhang, "Diagnosis of incipient faults in nonlinear analog circuits", Metrol. Meas. Syst., vol. 19, no. 2, pp. 203-218, 2012. DOI: 10.2478/v10178-012-0018-7.

[11] D. Grzechca, "Soft fault clustering in analog electronic circuits with the use of self organizing neural network", Metrol. Meas. Syst., vol. 18, no. 4, pp. 555-568, 2011. DOI: 10.2478/v10178-011-0054-8.

[12] A. Vasan, B. Long, M. Pecht, "Experimental validation of LS-SVM based fault identification in analog circuits using frequency features", Lect. Notes Mech. Eng., 2014. DOI: 10.1007/978-1-4471-4993-4_54.

[13] S. Temich, L. Chruszczyk, D. Grzechca, "Identification of the specification parameters for a voltage controlled oscillator using an artificial neural network with a genetic algorithm", Elektronika ir Elektrotechnika, vol. 24, no. 6, pp. 42-49, 2018. DOI: 10.5755/j01.eie.24.6.20945.

[14] S. P. Karthi, M. Shanthi, M. C. Bhuvaneswari, "Parametric fault diagnosis in analog circuit using genetic algorithm", in Int. Conf. Green Computing Communication and Electrical Engineering (ICGCCEE 2014), Coimbatore, India, 2014, pp. 1-5. DOI: 10.1109/ICGCCEE.2014.6921410.

[15] T. Golonek, J. Rutkowski, "Genetic-algorithm-based method for optimal analog test points selection", IEEE Trans. Circuits Syst. II Express Briefs, vol. 54, no. 2, pp. 117-121, 2007. DOI: 10.1109/TCSII.2006.884112.

[16] D. E. Grzechca, "Construction of an expert system based on fuzzy logic for diagnosis of analog electronic circuits", Int. J. Electron. Telecommun., vol. 61, no. 1, pp. 77-82, 2015. DOI: 10.1515/eletel2015-0010.

[17] R. J. Baker, CMOS: Circuit Design, Layout, and Simulation. Hoboken, NJ, USA: John Wiley \& Sons, Inc., 2010. DOI 10.1002/9780470891179.

[18] H. Dadhich, V. Maurya, K. Verma, S. Jaiswal, "Design and analysis of different type of charge pump using CMOS technology", in Int Conf. Advances in Computing, Communications and Informatics 
(ICACCI 2016), Jaipur, India, 2016, pp. 294-298. DOI: 10.1109/ICACCI.2016.7732062.

[19] S. R. Anumula, "Fully integrated CMOS charge pump design", M.S thesis, Dept. Electrical and Computer Engineering, Univ. Texas at Austin, Austin, Texas, USA, p. 63. [Online]. Available: repositories.lib.utexas.edu/handle/2152/ETD-UT-2010-08-1852

[20] T. Hillebrand, N. Hellwege, N. Heidmann, S. Paul, D. PetersDrolshagen, "Charge-based stochastic aging analysis of CMOS circuits", in IEEE International Integrated Reliability Workshop (IIRW 2015), South Lake Tahoe, CA, USA, 2015, pp. 126-129. DOI: 10.1109/IIRW.2015.7437084.

[21] S. Johnson, "Design for a discrete charge pump", Texas Instruments. [Online]. Available: http://www.ti.com/lit/an/slva398a/slva398a.pdf

[22] C. L. Singh, A. J. Gogoi, C. Anandini, K. L. Baishnab, "Low-noise CMOS differential-amplifier design using automated-design methodology", in Devices for Integrated Circuit (DevIC 2017), Kalyani, India, 2017, pp. 326-330. DOI: 10.1109/DEVIC.2017.8073962.
[23] Z. Liu, S. K. Chaganti, D. Chen, "Improving time-efficiency of faultcoverage simulation for MOS analog circuit", IEEE Trans. Circuits and Systems I: Regular Papers, vol. 65, no. 5, pp. 1664-1674, 2018 DOI: $10.1109 /$ TCSI.2017.2751561

[24] T. H. Morshed et al., "BSIM4v4. 7 MOSFET model", Dept Elect Eng Comput Sci Univ Calif. Berkeley Berkeley CA USA Tech Rep, 2011 [Online]. Available: http://cmosedu.com/cmos1/BSIM4_manual.pdf

[25] Control System Toolbox Documentation. [Online]. Available: https://www.mathworks.com/help/control

[26] J. Ardizzoni, "High-speed time-domain measurements - practical tips for improvement", p. 6, 2007. [Online]. Available: https://www.analog.com/en/analog-dialogue/articles/hgh-speed-timedomain-measurements.html

[27] K. Price, R. M. Storn, J. A. Lampinen, Differential evolution: a practical approach to global optimization. Berlin Heidelberg: Springer-Verlag, 2005. DOI: 10.1007/3-540-31306-0.

[28] A. Sen, M. Srivastava, Regression Analysis. New York, NY: Springer New York, 1990. DOI: 10.1007/978-1-4612-4470-7. 